\title{
Moduli of Super Riemann Surfaces
}

\author{
Claude LeBrun ${ }^{1, \star}$ and Mitchell Rothstein ${ }^{2, \star \star}$ \\ ${ }^{1}$ Department of Mathematics, State University of New York, Stony Brook, NY 11794, USA \\ ${ }^{2}$ School of Mathematics, Institute for Advanced Study, Princeton, NJ 08540, USA
}

\begin{abstract}
The basic properties of super Riemann surfaces are presented, and their supermoduli spaces are constructed, in a manner suitable for the application of algebro-geometric techniques to string theory.
\end{abstract}

\section{Introduction}

Super Riemann surfaces were introduced by Friedan $[\mathrm{Fr}]$ as the correct supersymmetric analogue of a Riemann surface, and the supermoduli space plays a role in superstring theory analogous to the role of moduli space in bosonic string theory. In this paper we will provide a description of supermoduli space in precise sheaf-theoretic terms, following the classical lines of Kodaira-Spencer deformation theory.

We will in fact construct the supermoduli spaces for super Riemann surfaces with level- $n$ structure, $n \geqq 3$. The choice of $n=3$ as the lower limit is dictated by the fact that 3 is the least of all integers $n$ such that an automorphism of a Riemann surface $(\Sigma, \mathcal{O})$ inducing the identity on $H^{1}(\Sigma, \mathbf{Z} / n \mathbf{Z})$ is itself the identity map $[\mathrm{F}-\mathrm{K}]$. It follows that the reduced space of the level- $n$ supermoduli space is nonsingular. Nevertheless, the supermoduli spaces are orbifolds, so to speak, in the "odd" directions. Each is locally the quotient of a supermanifold by the canonical automorphism which sends any superfunction $f$ of definite parity $|f|$ to $(-1)^{|f|} f$. The construction calls attention to the topological problem of determining, for each value of $n$, whether the level- $n$ supermoduli space is a global orbifold. This is equivalent to asking whether the spin structures on the fibers of the universal curve over the reduced space may be fitted together to form a square root of the canonical bundle of that curve. Some remarks about this problem are given in the appendix.

One may also consider super Riemann surfaces with a homotopy marking. The corresponding supermoduli space is a global orbifold. It is the quotient, by the

$\star$ Supported in part by NSF Grant No. DMS- 8704401

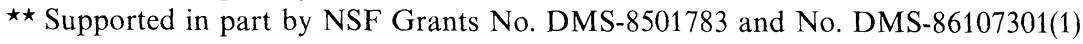


canonical automorphism, of super Teichmüller space. For a given genus $g$, let $\widetilde{T}$ denote the $4^{g}$-fold cover of Teichmüller space corresponding to all possible spin structures, and let $\left(U, \kappa^{1 / 2}\right) \rightarrow \widetilde{T}$ denote the universal curve with its canonical square root of $\kappa$. Then super Teichmüller space is $\left(\widetilde{T}, \wedge \pi_{*}^{0}\left(\kappa^{3 / 2}\right)\right)$, where $\pi_{*}^{0}\left(\kappa^{3 / 2}\right)$ is the $0^{\text {th }}$ direct image sheaf of $\kappa^{3 / 2}$. In particular, super Teichmüller space, and all the supermoduli spaces, are superspaces of dimension $(3 g-3,2 g-2)$ (see $[\mathrm{Fr}, \mathrm{C}-\mathrm{R}]$ ).

All of the supermoduli spaces carry an action of the mapping class group, and one may obtain a "true" supermoduli space as the quotient of super Teichmüller space by this action. The supermoduli spaces with level- $n$ structure are smooth finite branched covers of the full quotient, and therefore provide an appropriate setting for superstring theory.

\section{Super Riemann Surfaces}

First recall the definition of a supermanifold. Let $(X, \mathscr{A})$ be a $\mathbf{Z}_{2}$-gradedcommutative ringed space over $\mathbf{C}$. Let $\mathscr{N} \subset \mathscr{A}$ be the ideal of nilpotents. $(X, \mathscr{A})$ is a complex supermanifold if

1.1. $(X, \mathscr{A} / \mathscr{N})$ is a complex manifold.

1.2. $\mathscr{N} / \mathscr{N}^{2}$ is locally free over $\mathscr{A} / \mathscr{N}$ and $\mathscr{A}$ is locally isomorphic to $\wedge_{(\mathscr{A} / \mathscr{N})}\left(\mathscr{N} / \mathscr{N}^{2}\right)$.

For example, if $(X, \mathcal{O})$ is a complex manifold and $\mathscr{E}$ is a locally free sheaf of $(0$-modules, then $(X, \wedge \mathscr{E})$ is a supermanifold. Not all supermanifolds are of this form. For a general discussion of this point, see $[\mathrm{E}-\mathrm{L}, \mathrm{R}]$. A map $(X, \mathscr{A}) \stackrel{\pi}{\longrightarrow}(Y, \mathscr{B})$ between supermanifolds is a pair consisting of a reduced map $X \stackrel{\pi_{\text {red }}}{\longrightarrow} Y$ and a homomorphism $\pi_{\text {red }}^{-1}(\mathscr{B}) \stackrel{\pi^{*}}{\longrightarrow} \mathscr{A}$ of $\mathbf{Z}_{2}$-graded sheaves of $\mathbf{C}$-algebras. A family of compact supermanifolds is a map $(X, \mathscr{A}) \stackrel{\pi}{\longrightarrow}(Y, \mathscr{B})$ such that $\pi_{\text {red }}$ is proper and $\pi$ is submersive. The dimension of a supermanifold $(X, \mathscr{A})$ is the pair $(\operatorname{dim} X$, $\left.\operatorname{rank}\left(\mathscr{N} / \mathscr{N}^{2}\right)\right)$. The relative dimension of a family is the difference of the dimensions of the domain and range. Given a family $(X, \mathscr{A}) \stackrel{\pi}{\longrightarrow}(Y, \mathscr{B})$ and a point $p \in Y$, the fiber of the family at $p$ is the supermanifold $\pi^{-1}(p)=\left(\pi_{\text {red }}^{-1}(p), \mathscr{A} / \mathscr{I}_{p}\right)$, where $\mathscr{I}_{p} \subset \mathscr{A}$ is the ideal generated by $\pi^{*}\left(\pi^{-1}\left(\mathscr{M}_{p}\right)\right)$ and $\mathscr{M}_{p} \subset \mathscr{B}$ is the maximal ideal at $p$. The dimension of $\pi^{-1}(p)$ equals the relative dimension of the family. Let us denote supermanifolds by barred letters $\bar{X}, \bar{Y}$, etc., and let $\bar{X}$ stand for $\left(X, \mathscr{A}_{\bar{X}}\right)$. The tangent sheaf of $\bar{X}$ is the sheaf of graded derivations, $\operatorname{Der}\left(\mathscr{A}_{\bar{X}}\right)$, which we abbreviate by $\operatorname{Der}(\bar{X})$. If $\bar{X} \stackrel{\pi}{\longrightarrow} \bar{Y}$ is a family, the relative tangent sheaf is the sheaf $\operatorname{Der}_{\text {rel }}(\bar{X})$ $C \operatorname{Der}(\bar{X})$ consisting of derivations which annihilate $\pi^{*}\left(\mathscr{A}_{\bar{Y}}\right) . \operatorname{Der}_{\text {rel }}(\bar{X})$ is locally free, with rank equal to the relative dimension. If $\bar{X} \stackrel{\pi}{\longrightarrow} \bar{Y}$ has relative dimension $(p, q)$ and $z^{1}, \ldots, z^{p}, \eta^{1}, \ldots, \eta^{q}$ are sections of $\mathscr{A}_{\bar{X}}$ (with the $z$ 's even and the $\eta^{\prime}$ 's odd), such that $d z^{1}, \ldots, d z^{p}, \ldots, d \eta^{1}, \ldots, d \eta^{q}$ are a basis for $\operatorname{Der}_{\mathrm{rel}}^{*}(\bar{X})$, we call $(\mathbf{z}, \boldsymbol{\eta})$ a relative coordinate system. Then $\frac{\partial}{\partial z^{1}}, \ldots, \frac{\partial}{\partial z^{p}}, \frac{\partial}{\partial \eta^{1}}, \ldots, \frac{\partial}{\partial \eta^{q}}$ are defined to be the dual basis for
$\operatorname{Der}_{\text {rel }}(\bar{X})$.

Definition 1.1. A super Riemann surface over the supermanifold $\bar{Y}$ is a family of compact supermanifolds $\bar{X} \stackrel{\pi}{\longrightarrow} \bar{Y}$, of relative dimension $(1,1)$, together with a $(0,1)$ - 
dimensional subsheaf $\mathscr{D} \subset \operatorname{Der}_{\text {rel }}(\bar{X})$, such that the Lie bracket

$$
\mathscr{D}^{2} \stackrel{[,]}{\longrightarrow} \operatorname{Der}_{\text {rel }}(\bar{X}) / \mathscr{D}
$$

is an isomorphism. $\mathscr{D}^{2}$ denotes the tensor product $\mathscr{D} \otimes_{\mathscr{A}} \mathscr{D}$. A super Riemann surface is also called a SUSY-curve [M].

This definition connects with the standard definition in the physics literature by the following lemma.

Lemma 1.2. If $(\bar{X} \stackrel{\pi}{\longrightarrow} \bar{Y} ; \mathscr{D})$ is a super Riemann surface, one may cover $\bar{X}$ by relative coordinate systems $(z, \eta)$ such that $\mathscr{D}$ is generated by

$$
\frac{\partial}{\partial \eta}+\eta \frac{\partial}{\partial z}
$$

Proof. Let $(w, \lambda)$ be a relative coordinate system. Since $\mathscr{D}$ is locally free of rank $(0,1)$, it has a generator of the form $\frac{\partial}{\partial \lambda}+a \frac{\partial}{\partial w}$, with $a$ odd. Since $\mathscr{D}^{2} \stackrel{[,]}{\longrightarrow} \operatorname{Der}_{\text {rel }}(\bar{X}) / \mathscr{D}$ is an isomorphism, $\frac{\partial a}{\partial \lambda}$ must be invertible.

Introduce a coordinate system $(z, \eta)$ with $\eta=\lambda$. Then

$$
\frac{\partial}{\partial \lambda}+a \frac{\partial}{\partial w}=\frac{\partial}{\partial \eta}+\left(a \frac{\partial z}{\partial w}+\frac{\partial z}{\partial \lambda}\right) \frac{\partial}{\partial z} .
$$

Thus we must solve $a \frac{\partial z}{\partial w}+\frac{\partial z}{\partial \lambda}=\lambda$. Expand $z$ and $a$ as power series (first order) in $\lambda$ :

$$
z=\lambda z_{1}+z_{2}, \quad a=a_{1}+\lambda a_{2} .
$$

We obtain two equations:

$$
z_{1}+a_{1} \frac{\partial z_{2}}{\partial w}=0, \quad a_{2} \frac{\partial z_{2}}{\partial w}+a_{1} \frac{\partial z_{1}}{\partial w}=1
$$

Since $a_{2}$ is invertible, it is easy to see that this system has a solution.

Call a relative coordinate system $(z, \eta)$ canonical if $\mathscr{D}$ is generated by $\frac{\partial}{\partial \eta}+\eta \frac{\partial}{\partial z}$.

Note that by Lemma 1.2, one may define a super Riemann surface to be a family of $(1,1)$-dimensional supermanifolds together with an atlas of relative coordinate systems $\left(z_{\alpha}, \eta_{\alpha}\right)$ such that on the overlap of any two coordinate systems $\alpha$ and $\beta, \frac{\partial}{\partial \eta_{\alpha}}+\eta_{\alpha} \frac{\partial}{\partial z_{\alpha}}$ is a superfunction multiple of $\frac{\partial}{\partial \eta_{\beta}}+\eta_{\beta} \frac{\partial}{\partial z_{\beta}}$. This is the definition in $[\mathrm{Fr}]$.

Example. Super Riemann surfaces over a reduced base. Let $X \rightarrow Y$ be an ordinary complex curve over a complex manifold $Y$, and let $\kappa$ be the relative cotangent sheaf on $X$. If $\bar{X}=(X, \mathscr{A}) \rightarrow Y$ is a family of supermanifolds with relative dimension $(1,1)$, then $\mathscr{A}$ is canonically $\wedge(l)$ for some line bundle $l$. Indeed $\mathcal{O}_{X}=\mathscr{A}_{0}$ and $l=\mathscr{A}_{1}$. If $\bar{X}$ admits a superconformal structure $\mathscr{D}$, then for any $D \in \mathscr{D}, f \in \mathcal{O}_{X}$ and $\eta \in l, D(f \eta)$ 
$=D f \cdot \eta+f D \eta=f D \eta$, since $D f$ is odd and therefore of order $\eta$. Then $\mathscr{D}=l^{-1}$, and the bracket operation on $\mathscr{D}$ makes $l$ a square root of $\kappa$. Furthermore, $\left(X, \wedge \kappa^{1 / 2}\right)$ always admits a superconformal structure. Namely, if $(z, \eta)$ is a relative coordinate system for $\wedge \kappa^{1 / 2}$ such that $\eta \otimes \eta=d z$, then take $(z, \eta)$ as a canonical coordinate system. Thus a super Riemann surface over a reduced base is simply a family of Riemann surfaces $X \rightarrow Y$ together with a choice of $\kappa^{1 / 2}$.

\section{The Moduli Problem}

The goal is to find a universal super Riemann surface $(\bar{X} \rightarrow \bar{Y} ; \mathscr{D})$, meaning roughly that all super Riemann surfaces are obtained by maps into $\bar{Y}$. This makes sense only after a suitable notion of equivalence is given. First introduce a notion of marking. For a given genus $g$, fix a smooth surface $\Sigma$ of genus $g$. Then the family of smooth surfaces $X \rightarrow Y$ may be regarded as an associated fiber bundle of a principal Diff $\Sigma$ bundle over $X$, and a marking is simply a reduction of the structure group. We will consider two types of marking.

1. Homotopy marking, i.e., a reduction of the structure group from $\operatorname{Diff} \Sigma$ to $\operatorname{Diff}_{0} \Sigma$, where Diff ${ }_{0} \Sigma$ is the identity component of Diff $\Sigma$.

2. Level- $n$ structure, i.e., a reduction of the structure group from $\operatorname{Diff} \Sigma$ to Diff $_{n} \Sigma$, where $\operatorname{Diff}_{n} \Sigma$ is the kernel of the natural map

$$
\operatorname{Diff} \Sigma \rightarrow \operatorname{Aut}\left(H^{1}\left(\Sigma, \mathbf{Z}_{n}\right)\right) \text {. }
$$

If $\bar{X} \stackrel{\pi}{\longrightarrow} \bar{Y}$ is a family and $\bar{Z} \stackrel{\alpha}{\longrightarrow} \bar{Y}$ is a map, one obtains a family $\alpha^{*}(\bar{X}) \stackrel{\alpha^{*} \pi}{\longrightarrow} \bar{Z}$, called the pullback, defined as follows. Set

$$
\alpha^{*}(X)=\{(z, x) \in Z \times X \mid \alpha(z)=\pi(x)\},
$$

and set

$$
\mathscr{A}_{\alpha^{*}(\bar{X})}=\mathscr{A}_{\overline{\mathrm{Z}}} \hat{\otimes} \mathscr{A}_{\bar{X}} /\left(\left(\mathrm{id}_{\bar{Z}} \times \pi\right)^{*} \mathscr{I}\right),
$$

where $\mathscr{A}_{\bar{Z}} \hat{\otimes} \mathscr{A}_{\bar{X}}$ is the structure sheaf of $\bar{Z} \times \bar{X}$ and $\mathscr{I} C \mathscr{A}_{\bar{Z}} \hat{\otimes} \mathscr{A}_{\bar{Y}}$ is the ideal defining the graph of $\alpha$. If $\mathscr{D}$ is a superconformal structure on $\bar{X} \stackrel{\pi}{\longrightarrow} \bar{Y}$, then the pullback inherits a natural superconformal structure $\alpha^{*} \mathscr{D}$. If the underlying family of curves has a marking, one may also pull that back.

\section{The Canonical Automorphism and its Effect on the Moduli Problem}

Fix a type of marking. Then ask the question, does there exist a marked super Riemann surface $(\bar{X} \stackrel{\pi}{\longrightarrow} \bar{Y} ; \mathscr{D})$ such that given any marked super Riemann surface $\left(\bar{W} \stackrel{\sigma}{\longrightarrow} \bar{Z} ; \mathscr{D}^{\prime}\right)$, there is a unique map $\bar{Z} \stackrel{\alpha}{\longrightarrow} \bar{Y}$ such that $\left(\alpha^{*} \bar{X} \stackrel{\alpha^{*} \pi}{\longrightarrow} \bar{Z} ; \alpha^{*} \mathscr{D}\right)$ and $\left(\bar{W} \stackrel{\sigma}{\longrightarrow} \bar{Z} ; \mathscr{D}^{\prime}\right)$ are isomorphic over $\bar{Z}$, by an isomorphism which respects both the superconformal structure and the marking? The answer to this question is no, for the following reason. Define the canonical automorphism (of a supermanifold) to be the sheaf automorphism which sends any superfunction $f$ of definite parity $|f|$ to $(-1)^{|f|} f$. We denote this automorphism by $\Delta$. Then given any super Riemann 
surface $(\bar{X} \stackrel{\pi}{\longrightarrow} \bar{Y} ; \mathscr{D})$ and any map $\bar{Z} \stackrel{\alpha}{\longrightarrow} \bar{Y}, \alpha$ and $\Delta \circ \alpha$ induce equivalent super Riemann surfaces over $Z$.

The canonical automorphism has the following effect on the moduli problem. After constructing the reduced space of the supermoduli space by classical methods, one may use Kodaira-Spencer theory to construct pieces of the supermoduli space over small open sets in the reduced space. However, when trying to glue these pieces together on each pairwise intersection, one finds that there are two ways to do it, and no natural way to choose between them. As a result, the supermoduli space is defined only modulo $\Delta$. To say this more precisely, we introduce the following notions.

\section{Canonical Superorbifolds}

Define a quasimap between two supermanifolds $\bar{X}$ and $\bar{Y}$ to be an unordered pair of maps $\left\{\phi_{1}, \phi_{2}\right\} \subset \operatorname{Hom}(\bar{X}, \bar{Y})$ such that $\phi_{1}=\Delta \circ \phi_{2}$. Define a canonical superorbifold to be a set of data $\left(X,\left\{\overline{\mathscr{U}}_{\alpha}\right\}, \tau_{\alpha \beta}\right)$, where $X$ is a complex manifold, $\overline{\mathscr{U}}_{\alpha}=\left(\mathscr{U}_{\alpha}, \mathscr{A}_{\alpha}\right)$ is supermanifold defined on an open subset $\mathscr{U}_{\alpha} \subset X$, the sets $\mathscr{U}_{\alpha}$ cover $X$, and the $\tau_{\alpha \beta}$ are quasimaps

$$
\left.\left.\overline{\mathscr{U}}_{\alpha}\right|_{\mathscr{U}_{\alpha} \cap \mathscr{U}_{\beta}} \stackrel{\tau_{\alpha \beta}}{\longrightarrow} \overline{\mathscr{U}}_{\beta}\right|_{\mathscr{U}_{\alpha} \cap \mathscr{U}_{\beta}}
$$

which induce the identity on $X$ and satisfy the cocycle condition

$$
\tau_{\alpha \beta} \tau_{\beta \gamma} \tau_{\gamma \alpha}=\{\mathrm{id}, \Delta\} \text {. }
$$

Associated to any canonical superorbifold $\tilde{X}=\left(X,\left\{\overline{\mathscr{U}}_{\alpha}\right\}, \tau_{\alpha \beta}\right)$ is a class $\varepsilon \in H^{2}\left(X, \mathbf{Z}_{2}\right)$, obtained by choosing a representative, $\left.\hat{\tau}_{\alpha \beta} \in \operatorname{Hom}\left(\overline{\mathscr{U}}_{\alpha}, \overline{\mathscr{U}}_{\beta}\right)\right|_{\mathscr{U}_{\alpha} \cap \mathfrak{U}_{\beta}}$ for each $\tau_{\alpha \beta}$, and then defining $\varepsilon_{\alpha \beta \gamma}$ by

$$
\hat{\tau}_{\alpha \beta} \hat{\tau}_{\beta \gamma} \hat{\tau}_{\gamma \alpha}=\Delta^{\varepsilon_{\alpha \beta \gamma}} .
$$

If $\varepsilon$ vanishes, then we say that $\tilde{X}$ is a global superorbifold.

Notice that, while a canonical superorbifold is not a ringed space, there is a ringed space associated to it, for the even superfunctions are invariant under $\Delta$.

To construct the level- $n$ supermoduli space as a superorbifold, we proceed in two steps.

\section{Local Theory}

The first step in the local part of the moduli problem is to identify the sheaf of infinitesimal automorphisms. Let $(\bar{X} \stackrel{\pi}{\longrightarrow} \bar{Y} ; \mathscr{D})$ now be any super Riemann surface. Define sheaves $\mathscr{G}_{\pi}$ and $\mathscr{S}_{\pi}$ on $X$ :

$$
\mathscr{S}_{\pi}=\{\zeta \in \operatorname{Der}(\bar{X}) \mid[\zeta, \mathscr{D}] \subset \mathscr{D}\}, \quad \mathscr{G}_{\pi}=\mathscr{S}_{\pi} \cap \operatorname{Der} r_{\mathrm{rel}}(\bar{X}) .
$$

$\mathscr{G}_{\pi}$ is the infinitesimal automorphism sheaf of the super Riemann surface.

A simple but important lemma:

Lemma 2.1. $\mathscr{G}_{\pi} \simeq \mathscr{D}^{2}$ as sheaves of $\pi^{-1}\left(\mathscr{A}_{\bar{Y}}\right)$ modules.

Proof. Recall

$$
0 \rightarrow \mathscr{D} \rightarrow \operatorname{Der}_{\mathrm{rel}}(\bar{X}) \stackrel{q}{\longrightarrow} \mathscr{D}^{2} \rightarrow 0
$$


We claim that $\left.q\right|_{\mathscr{G}_{\pi}}$ is bijective. Let $(z, \eta)$ be canonical coordinates and set $D=\frac{\partial}{\partial \eta}+\eta \frac{\partial}{\partial z}$. Take $\zeta \in \mathscr{G}_{\pi}$ and set $\zeta=a \frac{\partial}{\partial z}+b D$. Then

$$
[D, \zeta]=(D a) \frac{\partial}{\partial z}-(-1)^{|\zeta|} b \frac{\partial}{\partial z}+(D b) D
$$

Thus

$$
\zeta \in \mathscr{G}_{\pi} \leftrightarrow b=(-1)^{|\zeta|} D a
$$

The result follows.

Example. If $\left(X \rightarrow Y, \kappa^{1 / 2}\right)$ is a super Riemann surface over a reduced base, then $\mathscr{D}^{n}$ has a generator in $\kappa^{-n / 2}$. Then

$$
\mathscr{D}^{n}=\kappa^{-n / 2} \otimes\left(\mathcal{O} \oplus \kappa^{1 / 2}\right)=\kappa^{-n / 2} \oplus \kappa^{\frac{1-n}{2}} .
$$

Thus over a reduced base, $\mathscr{G}_{\pi}=\kappa^{-1} \oplus \kappa^{-1 / 2}$.

Let $\pi_{*}^{i}$ denote the $i^{\text {th }}$ direct-image functor, i.e., for any sheaf $\mathscr{F}$ of abelian groups on $X, \pi_{*}^{i}(\mathscr{F})$ is the sheaf on $Y$ obtained by completing the presheaf

$$
\mathscr{U} \rightarrow H^{i}\left(\pi^{-1}(\mathscr{U}), \mathscr{F}\right) .
$$

Proposition 2.2. Let $(\bar{X} \stackrel{\pi}{\longrightarrow} \bar{Y} ; \mathscr{D})$ be a super Riemann surface of genus $g \geqq 2$. Then $\pi_{*}^{0}\left(\mathscr{G}_{\pi}\right)=0$.

Proof. Let $\mathscr{N} \subset \mathscr{A}_{\bar{Y}}$ denote the nilpotent ideal. Set

$$
\bar{Y}^{(n)}=\left(Y, \mathscr{A}_{\bar{Y}} / \mathscr{N}^{n+1}\right), \quad \bar{X}^{(n)}=\left(X, \mathscr{A}_{\bar{X}} /\left(\pi^{*}\left(\mathscr{N}^{n+1}\right)\right)\right) .
$$

Define $\mathscr{G}_{\pi}^{(n)} \subset \operatorname{Der}_{\text {rel }}\left(\bar{X}^{(n)}\right)$, by analogy with $\mathscr{G}_{\pi}$. Since the assertion is local, we may assume $\mathscr{A}_{\bar{Y}}=\wedge \mathscr{E}$, where $\mathscr{E}$ is a locally free sheaf of $\mathcal{O}_{\overline{\mathbf{Y}}}$-modules. Then we have

$$
0 \rightarrow \pi_{\mathrm{red}}^{-1}\left(\wedge^{n} \mathscr{E}\right) \otimes_{\pi_{\mathrm{red}}^{-1}\left(\mathcal{O}_{\bar{Y}}\right)} \mathscr{G}_{\pi}^{0} \rightarrow \mathscr{G}_{\pi}^{(n)} \rightarrow \mathscr{G}_{\pi}^{(n-1)} \rightarrow 0 .
$$

As stated in the example above, $\mathscr{G}_{\pi}^{0}=\kappa^{-1} \oplus \kappa^{-1 / 2}$. Thus $\left(\pi_{\text {red }}\right)_{*}^{0}\left(\mathscr{G}_{\pi}^{0}\right)=0$, and the proposition follows by induction on $n$.

Let $\bar{Z}$ be a supermanifold, and let $\left(W \stackrel{\sigma}{\longrightarrow} Z ; \kappa^{1 / 2}\right)$ be a super Riemann surface over the reduced space. We wish to characterize the super Riemann surfaces over $\bar{Z}$ which pull back to $\left(W \stackrel{\sigma}{\longrightarrow} Z ; \kappa^{1 / 2}\right)$. There is a characterization in terms on nonabelian sheaf cohomology, at least when $\bar{Z}$ is split. First declare two super Riemann surfaces $(\bar{W} \stackrel{\bar{\sigma}}{\longrightarrow} \bar{Z} ; \mathscr{D})$ and $\left(\bar{W}^{\prime} \stackrel{\bar{\sigma}^{\prime}}{\longrightarrow} \bar{Z}^{\prime} ; \mathscr{D}^{\prime}\right)$ extending $\left(W \stackrel{\sigma}{\longrightarrow} Z ; \kappa^{1 / 2}\right)$ to be equivalent if there exists a superconformal isomorphism

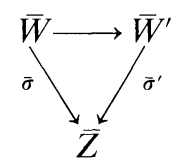

which restricts along $Z$ to $\sigma$. Now assume $\bar{Z}=(Z, \wedge \mathscr{E})$, where $\mathscr{E}$ is a sheaf of $\mathcal{O}_{Z}$-modules. Extend the family $W \stackrel{\sigma}{\longrightarrow} Z$ trivially in the $\mathscr{E}$-directions to obtain a 
super Riemann surface

$$
\bar{W}=\left(W, \wedge \mathscr{E} \otimes \wedge \kappa^{1 / 2}\right) \rightarrow(Z, \wedge \mathscr{E})
$$

$\left[\wedge \mathscr{E} \otimes \wedge \kappa^{1 / 2}\right.$ is an abbreviation for $\sigma^{-1}(\wedge \mathscr{E}) \otimes_{\sigma^{-1}\left(\mathcal{O}_{Z}\right)} \wedge \kappa^{1 / 2}$. Let $\mathscr{N}$ denote the nilpotent ideal of $\wedge \mathscr{E}$. Over $W$ one has a sheaf of nilpotent Lie superalgebras $\mathscr{N} \otimes \mathscr{G}_{\sigma}$. Let $G$ denote the sheaf of groups

$$
G=\exp \left(\left(\mathscr{N} \otimes \mathscr{G}_{\sigma}\right)_{\mathrm{even}}\right) .
$$

Lemma 2.3. The set of equivalence classes of super Riemann surfaces $(\bar{W} \stackrel{\bar{\sigma}}{\longrightarrow} \bar{Z} ; \mathscr{D})$ extending $\left(W \stackrel{\sigma}{\longrightarrow} Z ; \kappa^{1 / 2}\right)$ is in natural $1-1$ correspondence with $H^{1}(W, G)$.

Proof. Given a neighborhood $\mathscr{U} \subset W$, consider a super-Riemann-surface automorphism

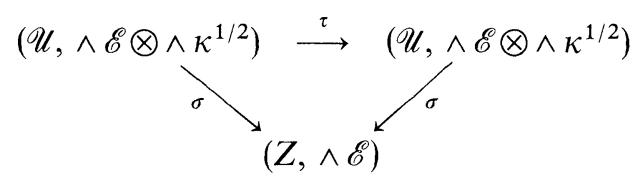

which is trivial $\bmod \mathscr{N}$. If $(z, \eta)$ is a relative coordinate system, then

$$
\tau(z, \eta)=(z+\delta z, \eta+\delta \eta)
$$

where $\delta z$ and $\delta \eta$ lie in $\mathscr{N} \otimes \wedge \kappa^{1 / 2}$. It follows that $1-\tau$ is nilpotent, so that one may define $\log (\tau)$ as a power series about the identity. Thus $\tau=\exp (\zeta)$, where $\zeta$ is a section of

$$
\left(\mathcal{N} \otimes D e r_{\text {rel }}\left(\wedge \kappa^{1 / 2}\right)\right)_{\text {even }}
$$

Since the superconformal structure is preserved, $\zeta$ lies in $\mathscr{N} \otimes \mathscr{G}_{\sigma^{.}}$If $\left(\bar{W}^{\prime} \stackrel{\bar{\sigma}}{\longrightarrow} \bar{Z} ; \mathscr{D}\right)$ is a super Riemann surface which pulls back to $\left(W \stackrel{\sigma}{\longrightarrow} Z ; \kappa^{1 / 2}\right)$, then $\bar{W}^{\prime}$ is obtained by gluing together local bits of $\bar{W}$ via a cocycle of automorphisms of the type just described. This proves the lemma.

Now take $\bar{Z}$ as above, and assume furthermore that $Z$ is Stein.

Lemma 2.4. Let $\mathscr{N}$ denote the nilpotent ideal in $\wedge \mathscr{E}$ and let $\bar{Z}^{(n)}=\left(Z, \wedge \mathscr{E} / \mathscr{N}^{n+1}\right)$. Then

1. Any super Riemann surface $\left(\bar{X} \stackrel{\pi}{\longrightarrow} \bar{Z}^{(n)} ; \mathscr{D}\right)$ over $\bar{Z}^{(n)}$ may be extended to a super Riemann surface over $\bar{Z}^{(n+1)}$.

2. The space of equivalence classes of such extensions is naturally an affine space modelled on

$$
\Gamma\left(Z,\left(\wedge^{n} \mathscr{E} \otimes \pi_{*}^{1}\left(\mathscr{G}_{\pi_{\mathrm{red}}}\right)\right)_{\mathrm{even}}\right) .
$$

Remark. Though $\bar{Z}^{(n)}$ is not a supermanifold, there is no difficulty in defining the notion of super Riemann surface over $\bar{Z}^{(n)}$, in complete analogy with the definition given in Sect. 1.

Proof. Suppose

$$
1 \rightarrow A \rightarrow G_{1} \rightarrow G_{2} \rightarrow 1
$$


is an exact sequence of sheaves of groups over a space $X$, with $A$ central. Then by pushing the long exact sequence as far as it will go, one finds

1. There is a connecting map $H^{1}\left(X, G_{2}\right) \stackrel{\Delta_{1}}{\longrightarrow} H^{2}(X, A)$, such that

$$
H^{1}\left(X, G_{1}\right) \rightarrow H^{1}\left(X, G_{2}\right) \stackrel{\Delta_{1}}{\longrightarrow} H^{2}(X, A)
$$

is exact.

2. Given $\tau \in H^{1}\left(X, G_{2}\right)$, one may form a group $H_{\tau}^{0}\left(X, G_{2}\right)$. If $\tau$ is given by a 1-cocycle $\tau_{\alpha \beta}$, then $H_{\tau}^{0}\left(X, G_{2}\right)$ is the group of 0 -cochains $\left\{\varrho_{\alpha}\right\}$ satisfying $\varrho_{\alpha} \tau_{\alpha \beta} \varrho_{\beta}^{-1}=\tau_{\alpha \beta}$.

3. There is a connecting homomorphism $H_{\tau}^{0}\left(X, G_{2}\right) \stackrel{\Delta_{0}}{\longrightarrow} H^{1}(X, A)$, and if $\Delta_{1}(\tau)=0$, then $H^{1}(X, A)$ acts transitively on the fiber of $H^{1}\left(X, G_{1}\right) \rightarrow H^{1}\left(X, G_{2}\right)$ over $\tau$, with the kernel being the image of $\Delta_{0}$.

To apply this to our situation, let $\sigma=\pi_{\text {red }}$, and define $\mathscr{G}_{\sigma}^{(n+1)}$ as in the proof of Proposition 2.2. Then we have

$$
0 \rightarrow\left(\wedge^{n+1} \mathscr{E} \otimes \mathscr{G}_{\sigma}\right)_{\text {even }} \rightarrow \exp \left(\left(\mathscr{G}_{\sigma}^{(n+1)}\right)_{\text {even }}\right) \rightarrow \exp \left(\left(\mathscr{G}_{\sigma}^{(n)}\right)_{\text {even }}\right) \rightarrow 1 .
$$

Because $Z$ is Stein,

$$
H^{*}\left(X, \mathscr{G}_{\sigma}^{(n)}\right)=\Gamma\left(Z, \sigma_{*}^{\cdot}\left(\mathscr{G}_{\sigma}^{(n)}\right)\right) .
$$

The lemma now follows from points 1,2 , and 3. Indeed, $\Gamma\left(Z, \sigma_{*}^{2}\left(\mathscr{G}_{\sigma}^{(n)}\right)\right)$ vanishes, since the fibers of $\sigma$ are curves. This and point 1 prove the first assertion. To prove the second assertion, take $\tau \in H^{1}\left(X, \exp \left(\left(\mathscr{G}_{\sigma}^{(n)}\right)_{\text {even }}\right)\right.$. Since $\tau$ is a family, one may form its sheaf of infinitesimal automorphisms, $\mathscr{G}_{\tau}$. Then $H_{\tau}^{0}\left(X, \exp \left(\left(\mathscr{G}_{\sigma}^{(n)}\right)_{\text {even }}\right)\right)$ is precisely $H^{0}\left(X, \exp \left(\left(\mathscr{G}_{\tau},\right)_{\text {even }}\right)\right.$. By point $3, \Gamma\left(Z,\left(\wedge^{n} \mathscr{E} \otimes \sigma_{*}^{1}\left(\mathscr{G}_{\sigma}\right)\right)_{\text {even }}\right)$ acts transitively on the extensions of $\tau$, and the kernel of the action in $H^{0}\left(X, \exp \left(\left(\mathscr{G}_{\tau},\right)_{\text {even }}\right)\right.$. By Proposition 2.2, the latter vanishes, so the action of $\Gamma\left(Z,\left(\wedge^{n} \mathscr{E} \otimes \sigma_{*}^{1}\left(\mathscr{G}_{\sigma}\right)\right)_{\text {even }}\right)$ on $H^{1}\left(X, \exp \left(\left(\mathscr{G}_{\sigma}^{(n)}\right)_{\text {even }}\right)\right)$ is faithful.

The next ingredient is the Kodaira-Spencer map. Given any super Riemann surface $(\bar{X} \stackrel{\pi}{\longrightarrow} \bar{Y} ; \mathscr{D})$, the differential of $\pi$ defines an exact sequence

$$
0 \rightarrow \mathscr{G}_{\pi} \rightarrow \mathscr{S}_{\pi} \stackrel{d \pi}{\longrightarrow} \pi^{-1}(\operatorname{Der}(\bar{Y})) \rightarrow 0 .
$$

The Kodaira-Spencer map is the connecting homomorphism

$$
\operatorname{Der}(\bar{Y}) \stackrel{k s(\pi)}{\longrightarrow} \pi_{1}^{*}\left(\mathscr{G}_{\pi}\right) .
$$

The Kodaira-Spencer map behaves naturally with respect to pullback, in the following sense. Take a split supermanifold $\bar{Z}=(Z, \wedge \mathscr{E})$ as before, and take $Z$ to be Stein. Define $\bar{Z}^{(n)}$ as in Lemma 2.4. Let $\left(W \stackrel{\sigma}{\longrightarrow} Z ; \kappa^{1 / 2}\right)$ be a super Riemann surface over the reduced space, and define

$$
\begin{aligned}
F^{n}= & \{\text { equivalence classes of super Riemann surfaces } \\
& \text { over } \left.\bar{Z}^{(n)} \text { which restrict to } \sigma\right\} .
\end{aligned}
$$

We have seen that there is an action of $\Gamma\left(Z,\left(\wedge^{n} \mathscr{E} \otimes \sigma_{*}^{1}\left(\mathscr{G}_{\sigma}\right)\right)_{\text {even }}\right)$ on $F^{n}$. 
On the other hand, let $(\bar{X} \stackrel{\pi}{\longrightarrow} \bar{Y} ; \mathscr{D})$ be a super Riemann surface and let $\bar{Z}^{(n)} \stackrel{h}{\longrightarrow} \bar{Y}$ be a map. Let $Z \stackrel{f}{\longrightarrow} Y$ be the reduced map of $h$, so that $h$ consists of the pair $\left(f, h^{*}\right)$, where $h^{*}$ is a homomorphism $f^{-1}\left(\mathscr{A}_{\bar{Y}}\right) \stackrel{h^{*}}{\longrightarrow} \wedge^{(n)} \mathscr{E}$. If $\zeta$ is a section over $Z$ of $\left(\wedge^{n} \mathscr{E} \otimes_{\mathcal{O}_{\bar{Y}}} f^{*}(\operatorname{Der}(\bar{Y}))\right)_{\text {even }}\left(=\operatorname{Der}_{\text {even }}\left(f^{-1}\left(\mathscr{A}_{\bar{Y}}\right), \wedge^{n} \mathscr{E}\right)\right)$, then $\zeta+h^{*}$ defines a new map. Thus if we set

$$
M^{n}=\left\{\operatorname{maps} \bar{Z}^{(n)} \rightarrow \bar{Y} \text { which restrict to } f\right\},
$$

we find that there is an action of $\Gamma\left(Z,\left(\wedge^{n} \mathscr{E} \otimes f^{*}(\operatorname{Der}(\bar{Y}))\right)_{\text {even }}\right)$ on $M^{n}$.

Now assume that $\left(W \stackrel{\sigma}{\longrightarrow} Z ; \kappa^{1 / 2}\right)$ is the pullback of $(\bar{X} \stackrel{\pi}{\longrightarrow} \bar{Y} ; \mathscr{D})$ via $f$. Then the Kodaira-Spencer map of $(\bar{X} \stackrel{\pi}{\longrightarrow} \bar{Y} ; \mathscr{D})$ gives us a map $f^{*}(\operatorname{Der}(\bar{Y})) \stackrel{k s(\pi)}{\longrightarrow} \sigma_{*}^{1}\left(\mathscr{G}_{\sigma}\right)$, which induces a map which we will again call $k s(\pi)$,

$$
\Gamma\left(Z,\left(\wedge^{n} \mathscr{E} \otimes f^{*}(\operatorname{Der}(\bar{Y}))\right)_{\mathrm{even}}\right) \stackrel{k s(\pi)}{\longrightarrow} \Gamma\left(Z,\left(\wedge^{n} \mathscr{E} \otimes \sigma_{*}^{1}\left(\mathscr{G}_{\sigma}\right)\right)_{\mathrm{even}}\right) .
$$

Lemma 2.5. Let

$$
M^{n} \stackrel{p^{n}}{\longrightarrow} F^{n}
$$

denote the pullback map. Then for all

$$
\zeta \in \Gamma\left(Z,\left(\wedge^{n} \mathscr{E} \otimes f^{*}(\operatorname{Der}(\bar{Y}))\right)_{\mathrm{even}}\right),
$$

and all $h \in M^{n}$,

$$
p^{n}(h+\zeta)=p^{n}(h)+k s(\pi)(\zeta) .
$$

Proof. We may assume that $\bar{Y}$ is a coordinate neighborhood. Let $w^{1}, \ldots, w^{M}$ be supercoordinates on $\bar{Y}$. Cover $X$ by open sets $\mathscr{U}_{\alpha}$ equipped with canonical relative (super)coordinates $\left(u_{\alpha}^{1}, u_{\alpha}^{2}\right)$. Then express $\left(u_{\beta}^{1}, u_{\beta}^{2}\right)$ as a power series in $\left(\mathbf{w}, \mathbf{u}_{\alpha}\right)$ :

$$
u_{\beta}^{i}=\tau_{\alpha \beta}^{i}\left(\mathbf{w}, \mathbf{u}_{\alpha}\right) \text {. }
$$

If $h \in M^{n}$, then the super Riemann surface $p^{n}(h)$ admits relative coordinate systems $\left(\tilde{u}_{\alpha}^{1}, \tilde{u}_{\alpha}^{2}\right)$, related by

$$
\tilde{u}_{\beta}^{i}=\tau_{\alpha \beta}^{i}\left(h^{*}(\mathbf{w}), \mathbf{u}_{\alpha}\right)
$$

Take $\zeta \in \Gamma\left(Z,\left(\wedge^{n} \mathscr{E} \otimes f^{*}(\operatorname{Der}(\bar{Y}))\right)_{\text {even }}\right)$, and set $\zeta=g^{a} \frac{\partial}{\partial w^{a}}$. The $g^{a}$ 's are sections of $\wedge^{n} \mathscr{E} \cdot p^{n}(h+\zeta)$ will be characterized by transition functions

$$
\mathbf{u}_{\beta}=\tau_{\alpha \beta}\left(h^{*}\left(w^{1}\right)+\zeta\left(w^{1}\right), \ldots, h^{*}\left(w^{M}\right)+\zeta\left(w^{M}\right), \tilde{\mathbf{u}}_{\alpha}\right)=\tau_{\alpha \beta}+\left.g^{a} \frac{\partial \tau_{\alpha \beta}}{\partial \omega^{a}}\right|_{u_{\alpha}=\mathrm{const}} .
$$

Therefore $p^{n}(h+\zeta)-p^{n}(h)$ is the cohomology class

$$
\left[g^{a} \frac{\partial \tau_{\alpha \beta}}{\partial \omega^{a}} \frac{\partial}{\partial u_{\alpha}}\right] \in H^{1}\left(W, \wedge^{n} \mathscr{E} \otimes \mathscr{G}_{\sigma}\right),
$$

and this is precisely $k s(\pi)(\zeta)$. The lemma is proved.

We now arrive at the basic result. 
Theorem 2.6. Let $(\bar{X} \stackrel{\pi}{\longrightarrow} \bar{Y} ; \mathscr{D})$ be a super Riemann surface, let $Z \stackrel{f}{\longrightarrow} Y$ be a map between the reduced spaces, and let $\left(W \stackrel{\sigma}{\longrightarrow} Z ; \kappa^{1 / 2}\right)$ be the pullback of $\pi$ via $f$. Define $M^{n}$ and $F^{n}$ as above. Assume $\operatorname{Der}(\bar{Y}) \stackrel{k s(\pi)}{\longrightarrow} \pi_{*}^{1}\left(\mathscr{G}_{\pi}\right)$ restricts to an isomorphism along the reduced space, $Y$. Then for all $n$, the pullback map

$$
M^{n} \stackrel{p^{n}}{\longrightarrow} F^{n}
$$

is bijective.

Proof. First observe that by taking an open cover of $Z$, one may restrict to the case that $\bar{Z}=(Z, \wedge(\mathscr{E})), Z$ is Stein, and the range of $f$ lies in a coordinate neighborhood of $\bar{Y}$. One has restriction maps

$$
M^{n+1} \stackrel{a^{n+1}}{\longrightarrow} M^{n}
$$

and

$$
F^{n+1} \stackrel{b^{n+1}}{\longrightarrow} F^{n}
$$

and a commutative diagram

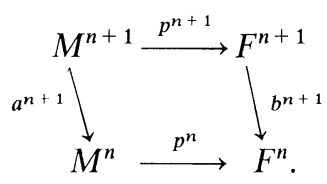

Observe that in the local situation, any map $h \in M^{n}$ lifts to a map at order $n+1$. Indeed, if $w^{1}, \ldots, w^{M}$ are supercoordinates on $\bar{Y}$, then $h$ gives the values of $h^{*}\left(w^{1}\right), \ldots, h^{*}\left(w^{M}\right)$ to order $n$, and we may extend these values arbitrarily to order $n+1$ to obtain a lift. Then $\left(a^{n+1}\right)^{-1}(h)$, being non-empty, is an affine space modelled on

$$
\Gamma\left(Z,\left(\wedge^{n+1} \mathscr{E} \otimes f^{*}(\operatorname{Der}(\bar{Y}))\right)_{\mathrm{even}}\right) .
$$

Now the Kodaira-Spencer map of $\pi$ is assumed to take $\Gamma\left(Z, \wedge^{n+1} \mathscr{E} \otimes f^{*}(\operatorname{Der}(\bar{Y}))\right)$ isomorphically onto $\Gamma\left(Z, \wedge^{n+1} \mathscr{E} \otimes \sigma_{*}^{1}\left(\mathscr{G}_{\sigma}\right)\right)$. Thus the fibers of $a^{n+1}$ and $b^{n+1}$ are affine spaces modelled on the same vector space. By Lemma $2.5, p^{n+1}$ intertwines the actions of this vector space, whence $p^{n+1}$ is bijective on each fiber. Then, by induction on $n, p^{n}$ is bijective for all $n$.

Remark. In proving Theorem 2.6, we used only the assumption that the KodairaSpencer map of $\pi$ restricted to an isomorphism along the reduced space. However, it follows from Proposition 2.2 that $\pi_{*}^{1}\left(\mathscr{G}_{\pi}\right)$ is always locally free. Therefore, under the hypotheses of Theorem 2.6, $k s(\pi)$ will in fact be an isomorphism over $\bar{Y}$.

Theorem 2.6 may be rephrased by saying that if $(\bar{X} \stackrel{\pi}{\longrightarrow} \bar{Y} ; \mathscr{D})$ is a super Riemann surface for which $k s(\pi)$ is an isomorphism, and $\left(\bar{W} \stackrel{\sigma}{\longrightarrow} \bar{Z} ; \mathscr{D}^{\prime}\right)$ is a super Riemann surface for which there is a map $Z \stackrel{\phi^{\prime}}{\longrightarrow} Y$ and a superconformal isomorphism

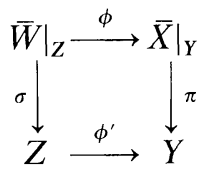


then there is a unique map $\bar{Z} \stackrel{\bar{\phi}^{\prime}}{\longrightarrow} \bar{Y}$ extending $\phi^{\prime}$ for which there exists an isomorphism

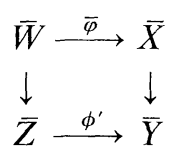

with $\bar{\psi}$ extending $\phi$. For the purposes of constructing the universal super Riemann surface, more control is needed over $\bar{\psi}$.

Theorem 2.7. Let $\bar{X} \stackrel{\pi}{\longrightarrow} \bar{Y}$ be any super Riemann surface for which $k s(\pi)$ is an isomorphism. Then the only superconformal isomorphisms

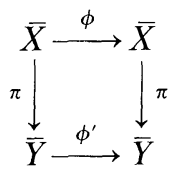

inducing the identity on $Y$ are id and the canonical automorphism, $\Delta$.

Proof. Let $\phi$ be such an isomorphism. Since $k s(\pi)$ is an isomorphism, the set of points $y \in Y$ for which $\pi_{\text {red }}^{-1}(y)$ admits an automorphism has positive codimension. Thus $\phi_{\text {red }}$ must be the identity. Now over $Y, \phi$ is an automorphism of $\kappa^{1 / 2}$ preserving the map $\kappa^{1 / 2} \otimes \kappa^{1 / 2} \rightarrow \kappa$. So along $Y, \phi=\mathrm{id}$ or $\Delta$. It remains to check that if $\phi=\mathrm{id}$ over $Y$, then $\phi=\mathrm{id}$. First observe that $\phi^{\prime}$ must be the identity, by Theorem 2.6. But if $\phi=\mathrm{id}$ along $Y$ and $\phi^{\prime}=\mathrm{id}$, then $\phi=\mathrm{id} \bmod$ nilpotents. It follows that $\phi=\exp (\zeta)$ for some $\zeta \in H^{0}\left(X, \mathscr{G}_{\pi}\right)$. By Proposition $2.2, \zeta=0$.

We complete our discussion of the local theory by establishing the local existence theorem for super Riemann surfaces.

Theorem 2.8. Let $\left(X \stackrel{\pi}{\longrightarrow} Y ; \kappa^{1 / 2}\right)$ be a super Riemann surface over a reduced base. Assume that $Y$ is Stein, and that $k s(\pi)$ (classical) is an isomorphism. Then there is a super Riemann surface $\bar{X} \stackrel{\pi}{\longrightarrow} \bar{Y}$, into which $\left(X \stackrel{\pi}{\longrightarrow} Y ; \kappa^{1 / 2}\right)$ may be imbedded, such that $k s(\bar{\pi})$ is an isomorphism.

Proof. We have $\mathscr{G}_{\pi} \simeq \kappa^{-1} \oplus \kappa^{-1 / 2}$. Let $\mathscr{E}=\pi_{*}^{1}\left(\kappa^{-1 / 2}\right)^{*}=\pi_{*}^{0}\left(\kappa^{3 / 2}\right)$. $\mathscr{E}$ is a locally free sheaf over $Y$, of rank $2 g-2$. Let $\bar{Y}=(Y, \wedge \mathscr{E})$. $\bar{Y}^{(1)}$ is simply $(Y, \mathcal{O} \oplus \mathscr{E})$, and there is an obvious super Riemann surface over $\bar{Y}^{(1)}$, namely the one which depends trivially on the $\mathscr{E}$-directions. Then by Lemma 2.4 , the super Riemann surfaces over $\bar{Y}^{(1)}$ are in natural $1-1$ correspondence with

$$
\Gamma\left(Y,\left(\mathscr{E} \otimes \pi_{*}^{1}\left(\mathscr{G}_{\pi}\right)\right)_{\mathrm{even}}\right)=\Gamma\left(Y, \mathscr{E} \otimes \pi_{*}^{1}\left(\mathscr{G}_{\pi}\right)_{\mathrm{odd}}\right)=\Gamma\left(Y, \mathscr{E} \otimes \mathscr{E}^{*}\right)=\Gamma(Y, \operatorname{End}(\mathscr{E})) .
$$

Thus there is another obvious super Riemann surface over $\bar{Y}^{(1)}$, namely the one corresponding to the identity section of End $(\mathscr{E})$. By Lemma 2.4 again, this super Riemann surface may be extended to a super Riemann surface $(\bar{X} \stackrel{\bar{\pi}}{\longrightarrow} \bar{Y} ; \mathscr{D})$. It remains to show that $k s(\bar{\pi})$ restricts to an isomorphism along the reduced space. The tangent sheaf of $\bar{Y}$ pulls back along $Y$ to $\Theta_{Y} \oplus \mathscr{E}^{*}$. In the first summand, $k s(\bar{\pi})$ is the usual Kodaira-Spencer map, which is an isomorphism. In the second summand, $k s(\bar{\pi})$ is, by construction, the identity map. This shows that $k s(\bar{\pi})$ is an isomorphism. 
Remark. A more explicit construction of a super Riemann surface for which $k s(\pi)$ is an isomorphism is given at the end of this section.

\section{Global Construction}

To complete the construction of the supermoduli space, it remains to solve an appropriate version of the moduli problem for reduced base spaces. We have already seen that a super Riemann surface over a reduced base is simply a curve $X \rightarrow Y$ together with a choice of $\kappa^{1 / 2}$. However, it is easy to see that there does not exist a curve $X \rightarrow Y$ with a level- $n$ structure and a choice of $\kappa^{1 / 2}$ which is universal. Indeed, suppose such an object existed. Then any curve $W \rightarrow Z$ with a choice of $\kappa^{1 / 2}$ would be induced by a unique map $Z \stackrel{\sigma}{\longrightarrow} Y$. However, one could modify $\kappa^{1 / 2}$ by a class $\omega \in H^{1}\left(Z, \mathbf{Z}_{2}\right)$. This produces no change in $\kappa^{1 / 2}$ locally in $Z$, and therefore it cannot change the map $\sigma$. So we see that the correct data for the moduli problem are not a curve with spin structure, but rather a curve together with a consistent choice of spin structure on each fiber.

Recall that the choice of $\kappa^{1 / 2}$ is strictly topological. Regard $X \rightarrow Y$ as a family of smooth surfaces, and let $T_{\text {rel }} X$ be the relative tangent bundle. Let $S_{\text {rel }} X$ $=\left(T_{\mathrm{rel}} X-\{0\}\right) / R^{+} . S_{\mathrm{rel}} X$ is a circle bundle over $X$. Let $\alpha \in H_{1}\left(S_{\mathrm{rel}} X, \mathbf{Z}_{2}\right)$ be the $\bmod 2$ class of any fiber $S^{1} \rightarrow S_{\text {rel }} X$. Then a choice of $\kappa^{1 / 2}$ corresponds to a choice of $\omega \in H^{1}\left(S_{\text {rel }} X, Z_{2}\right)$ such that $\omega[\alpha]$ is non-trivial. Associated to the fibration $S_{\mathrm{rel}} X \stackrel{\tilde{\pi}}{\longrightarrow} Y$ is a Leray spectral sequence

$$
E_{r}^{p, q} \Rightarrow \operatorname{Gr}\left(H^{p+q}\left(S_{\mathrm{rel}} X, \mathbf{Z}_{2}\right)\right) \quad \text { with } \quad E_{2}^{p, q}=H^{p}\left(Y, \tilde{\pi}_{*}^{q}\left(\mathbf{Z}_{2}\right)\right) .
$$

A choice of spin structure on each fiber of $X$ is simply an element $\sigma \in E_{2}^{0,1}$ $=H^{0}\left(Y, \tilde{\pi}_{*}^{1}\left(\mathbf{Z}_{2}\right)\right)$ which is non-trivial on the fiber class. The differential $d_{2}(\sigma) \in H^{2}\left(Y, \mathbf{Z}_{2}\right)$ must vanish for $\sigma$ to give rise to a choice of $\kappa^{1 / 2}$, and if $d_{2}(\sigma)=0$, the set of such choices is parametrized by $H^{1}\left(Y, \mathbf{Z}_{2}\right)$.

Define a fiberwise spin curve to be a curve $X \rightarrow Y$ together with a class $\sigma \in H^{0}\left(Y, \tilde{\pi}_{*}^{1}\left(\mathbf{Z}_{2}\right)\right)$ as described above.

Theorem 2.9. Universal objects exist in the following categories:

1. Fiberwise spin curves of a given genus $g \geqq 2$, with level- $n$ structure, $n \geqq 3$.

2. Fiberwise spin curves of a given genus $g \geqq 2$, with homotopy marking.

Proof. Let $(X \stackrel{\pi}{\longrightarrow} Y, \sigma)$ be a marked fiberwise spin curve of genus $g$, where the marking may be either a homotopy marking or a level- $n$ structure. The marking assigns to each point $y \in Y$ a preferred set of maps $\Gamma_{y} \subset \operatorname{Diff}\left(\pi^{-1}(y), \Sigma\right)$, where $\Sigma$ is the standard surface of genus $g$. If $\psi \in \Gamma_{y}$ is any such map, then $\psi$ transports the complex and spin structures on $\pi^{-1}(y)$ to complex and spin structures on $\Sigma$. Thus, if we let $\mathscr{C}$ be the space

$$
\mathscr{C}=\{\text { Complex structures on } \Sigma\} \times\{\text { Spin structures on } \Sigma\},
$$

then $\Gamma_{y}$ determines a point $\phi(y) \in \mathscr{C} / G$, where $G \subset \operatorname{Diff} \Sigma$ is the subgroup which determines the marking. It is essentially a classical result that $\mathscr{C} / G$ is a $3 g-3$ dimensional complex manifold, and the map $Y \stackrel{\phi}{\longrightarrow} \mathscr{C} / G$ is holomorphic. Indeed, in 
the case of the homotopy marking $\mathscr{C} / G$ is the trivial $4^{g}$-fold cover of Teichmüller space whose sheets correspond to the choices of $\kappa^{1 / 2}$ on the universal curve, and it well known that $\phi$ is holomorphic in this case [A, G, K-S]. Let us denote $\mathscr{C} / G$ for this case by $\widetilde{T}$. Then let $\Gamma$ denote the mapping class group, $\Gamma=\operatorname{Diff} \Sigma / \operatorname{Diff}_{0} \Sigma$, and let $\Gamma_{n}$ denote the kernel of the natural map

$$
\Gamma \rightarrow \operatorname{Aut}\left(H^{1}\left(\Sigma, \mathbf{Z}_{n}\right)\right) .
$$

For $n \geqq 3, \Gamma_{n}$ acts freely and properly discontinuously on $\widetilde{T},[\mathrm{~F}-\mathrm{K}]$, so that in the case of the level- $n$ structure, $\mathscr{C} / G=\widetilde{T} / \Gamma_{n}$ is again a smooth manifold. Over $\mathscr{C} / G$ is a curve $U=(\mathscr{C} \times \Sigma) / G$. It remains to give $U$ a fiberwise spin structure, such that $(X \stackrel{\pi}{\longrightarrow} Y, \sigma)$ is induced by $\phi$. For each point $(J, \omega) \in \mathscr{C}$, let $[J, \omega]$ be its equivalence class in $\mathscr{C} / G$. Then we get a diffeomorphism

$$
\begin{gathered}
\psi_{(J, \omega)}:\left.\Sigma \rightarrow U\right|_{[J, \omega]}, \\
\psi_{(J, \omega)}(x)=[J, \omega, x] .
\end{gathered}
$$

Given $g \in G$ we get a commutative diagram

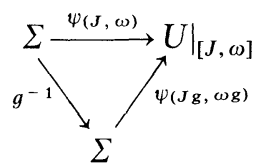

It follows that there is a well defined class

$$
\left.\hat{\sigma}_{[J, \omega]} \in H^{1}\left(S_{\text {rel }}\left(\left.U\right|_{[J, \omega]}\right), \mathbf{Z}_{2}\right)\right)
$$

given by

$$
\hat{\sigma}_{[J, \omega]}=\left(\psi_{(J, \omega)}^{-1}\right) *(\omega) .
$$

It is clear that $(U \rightarrow \mathscr{C} / G, \hat{\sigma})$ is the universal object.

We denote the universal level- $n$ fiberwise spin curve by $\left(U_{n} \stackrel{\pi}{\longrightarrow} M_{n} ; \sigma\right)$, and we denote the universal homotopy marked fiberwise spin curve by $(U \stackrel{\pi}{\longrightarrow} \widetilde{T} ; \sigma) . M_{n}$ is called level-n spinmoduli space.

\section{Construction of the Supermoduli Space and Universal Curve}

To complete the construction of the level- $n$ supermoduli space, let $\left\{\mathscr{U}_{\alpha}\right\}$ be a covering of $M_{n}$ by Stein open sets. On each $\pi^{-1}\left(\mathscr{U}_{\alpha}\right)$, choose $\kappa_{\alpha}^{1 / 2}$ compatible with $\sigma$. Let $\mathscr{E}_{\alpha}=\pi_{*}^{1}\left(\kappa_{\alpha}^{1 / 2}\right)$. Let $\overline{\mathscr{U}}_{\alpha}=\left(\mathscr{U}_{\alpha}, \wedge \mathscr{E}_{\alpha}\right)$. By Theorem 2.8 , we may construct super Riemann surfaces

$$
\left(\bar{X}_{\alpha} \stackrel{\pi_{\alpha}}{\longrightarrow} \overline{\mathscr{U}}_{\alpha} ; \mathscr{D}_{\alpha}\right)
$$

such that for all $\alpha, k s\left(\pi_{\alpha}\right)$ is an isomorphism. Theorems 2.6 and 2.7 together imply that on each intersection, $\pi^{-1}\left(\mathscr{U}_{\alpha} \cap \mathscr{U}_{\beta}\right)$, there are $t w o$ isomorphisms

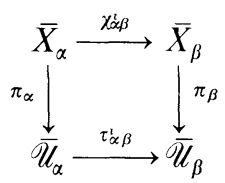


$i=1,2$. The pairs $\tilde{\chi}_{\alpha, \beta}=\left\{\chi_{\alpha \beta}^{1}, \chi_{\alpha \beta}^{2}\right\}$ and $\tilde{\tau}_{\alpha, \beta}=\left\{\tau_{\alpha \beta}^{1}, \tau_{\alpha \beta}^{2}\right\}$ are uniquely defined quasimaps, which therefore obey the cocycle condition. We now have canonical superorbifolds,

$$
\begin{gathered}
\tilde{X}_{n}=\left(U_{n},\left\{\bar{X}_{\alpha},\right\},\left\{\tilde{\chi}_{\alpha \beta}\right\}\right), \\
\tilde{M}=\left(M_{n},\left\{\overline{\mathscr{U}}_{\alpha}\right\},\left\{\tilde{\tau}_{\alpha \beta}\right\}\right) .
\end{gathered}
$$

$\tilde{M}$ is the level-n supermoduli space. The data $\left(\tilde{X},\left\{\pi_{\alpha}\right\},\left\{\mathscr{D}_{\alpha}\right\}\right)$, constitute the universal level-n super Riemann surface. Level- $n$ supermoduli space is universal in the following sense.

\section{Universal Property}

Given a supermanifold $\bar{Z}$ and a canonical superorbifold $\tilde{Y}=\left(Y,\left\{\overline{\mathscr{U}}_{\alpha}\right\}, \tau_{\alpha \beta}\right)$, a map $\bar{Z} \rightarrow \tilde{Y}$ is by definition a map $Z \stackrel{\varrho}{\longrightarrow} Y$ together with a collection of quasimaps

$$
\left.\bar{Z}\right|_{\varrho^{-1}\left(\mathscr{U}_{\alpha}\right)} \stackrel{\hat{\varrho}_{\alpha}}{\longrightarrow} \overline{\mathscr{U}}_{\alpha}
$$

such that for all $\alpha$ and $\beta$, the diagram

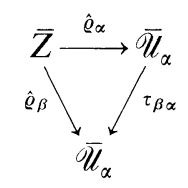

commutes.

Let $\bar{Z}$ be a supermanifold with $H^{1}\left(Z, \mathbf{Z}_{2}\right)=H^{2}\left(Z, \mathbf{Z}_{2}\right)=0$, and let $\tilde{\varrho}=\left(\varrho,\left\{\varrho_{\alpha}\right\}\right)$ be a map $\bar{Z} \rightarrow \tilde{M}_{n}$. We obtain a collection of super Riemann surfaces $\left(\hat{\varrho}_{\alpha}\right)^{*}\left(\bar{X}_{\alpha}\right)$ $\rightarrow \bar{Z} \mid \varrho^{-1}\left(\mathscr{U}_{\alpha}\right)$, and quasimaps between them. Since $H^{1}\left(Z, Z_{2}\right)=H^{2}\left(Z, \mathbf{Z}_{2}\right)=0$, there exists a unique way, up to equivalence, of resolving the $\mathbf{Z}_{2}$ ambiguities to fit these super Riemann surfaces together and construct a super Riemann surface over $\bar{Z}$. Denote this super Riemann surface (defined up to equivalence) by $\varrho^{*}(\widetilde{X})$. From Theorems 2.6 and 2.9 , we immediately obtain

Theorem 2.10. Given any supermanifold $\bar{Z}$ with $H^{1}\left(Z, Z_{2}\right)=H^{2}\left(Z, Z_{2}\right)=0$, and any super Riemann surface $(\bar{W} \rightarrow \bar{Z} ; \mathscr{D})$ with level-n structure, there is a unique map $\bar{Z} \stackrel{\hat{\varrho}}{\longrightarrow} \tilde{M}$ inducing the given level-n structure, such that $\tilde{\varrho}^{*}(\tilde{X})$ and $\bar{W}$ are superconformally equivalent.

\section{Super Teichmüller Space}

The constructions of the supermoduli space and universal super Riemann surface can of course be carried out for homotopy marked super Riemann surfaceš. Indeed, this is the easiest case. Since $\widetilde{T}$ is Stein [B-E], the local construction of the supermoduli space may be applied globally. The resulting base space, is therefore isomorphic to $\left(\widetilde{T}, \wedge \pi_{*}^{0}\left(\kappa^{3 / 2}\right)\right.$ ). (with the trivial open cover $\{\emptyset, T\}$ and the cocycle $\{\mathrm{id}, \Delta\}) .\left(\widetilde{T}, \wedge \pi_{*}^{0}\left(\kappa^{3 / 2}\right)\right)$ is called super Teichmüller space. 


\section{Uniqueness of Supermoduli Space}

Any supermanifold may be characterized up to isomorphism by data $(X, \tau)$, where $X$ is a complex manifold, and $\tau$ is a 1-cohomology class with coefficients in the automorphism sheaf of the supermanifold $\left(X, \wedge\left(\mathcal{O}^{\oplus m}\right)\right)$. Similarly, denoting this automorphism sheaf by $\mathscr{G}$, a canonical superorbifold over $X$ determines an isomorphism class $\tilde{\tau} \in H^{1}(X, \mathscr{G} / \Delta)$. The isomorphism class of supermoduli space is uniquely characterized by the universal property of Theorem 2.10 .

\section{Explicit Construction of Local Universal Families}

Finally, it is possible to be more explicit in the local construction of the supermoduli space. Again take the universal level- $n$ fiberwise spin curve $\left(U_{n} \stackrel{\pi}{\longrightarrow} M_{n} ; \sigma\right)$, take an open cover by Stein sets $\left\{\mathscr{U}_{\alpha}\right\}$, and on each $\pi^{-1}\left(\mathscr{U}_{\alpha}\right)$, choose $\kappa_{\alpha}^{1 / 2}$ compatible with $\sigma$. Fix $\alpha$ and set $\mathscr{U}=\mathscr{U}_{\alpha}$. Set $\mathscr{E}=\pi_{*}^{0}\left(\kappa_{\alpha}^{3 / 2}\right)$. Assume that $\left.U_{n}\right|_{\mathscr{U}}$ admits two disjoint relative divisors, $D_{1}$ and $D_{2}$. Let $\mathscr{V}_{i} \subset \pi^{-1}(\mathscr{U})$ denote the complement of $D_{i}, i=1,2$. Let $\theta^{1}, \ldots, \theta^{2 g-2}$ be a basis of sections of $\mathscr{E}$. The open cover $\left\{\mathscr{V}_{1}, \mathscr{V}_{2}\right\}$ computes the analytic sheaf cohomology of $\pi^{-1}(\mathscr{U})$, so we may choose sections $\phi_{1}, \ldots, \phi_{2 g-2} \in \Gamma\left(\mathscr{V}_{1} \cap \mathscr{V}_{2}, \kappa^{-1 / 2}\right)$ such that the cohomology classes

$$
\left[\phi_{1}\right], \ldots,\left[\phi_{2 g-2}\right] \in H^{1}\left(\left\{\mathscr{V}_{1}, \mathscr{V}_{2}\right\}, \kappa^{-1 / 2}\right)
$$

are dual to $\theta^{1}, \ldots, \theta^{2 g-2}$. Using now the isomorphism $\left(\mathscr{G}_{\pi}\right)_{\mathrm{odd}} \simeq \kappa^{-1 / 2}$, identify the sum $\theta^{i} \phi_{i}$ as a nilpotent derivation of $\pi^{-1}(\wedge \mathscr{E}) \otimes \wedge \kappa^{1 / 2}$. (Recall that if $\eta$ is a generator of $\kappa^{1 / 2}$ and $z$ is a relative coordinate such that $\eta^{2}=d z$, the identification of $\kappa^{-1 / 2}$ with $\mathscr{G}_{\pi}$ sends $\frac{\partial}{\partial \eta}$ to $\frac{\partial}{\partial \eta}-\eta \frac{\partial}{\partial z}$.) On each of $\mathscr{V}_{1}$ and $\mathscr{V}_{2}$, one has the super
Riemann surface

$$
\left(\left(\mathscr{V}_{i}, \pi^{-1}(\wedge \mathscr{E}) \otimes \wedge \kappa^{1 / 2}\right) \rightarrow(\mathscr{U}, \wedge \mathscr{E}) ; \mathscr{D}_{i}\right)
$$

obtained by extending trivially in the $\mathscr{E}$ directions. Now glue these super Riemann surfaces together over the intersection by the automorphism

$$
\exp \left(\theta^{i} \phi_{i}\right)
$$

Then the Kodaira-Spencer map of the resulting super Riemann surface is an isomorphism, and this gives an explicit local construction of the universal super Riemann surface.

For the purpose of understanding the cocycle $\bar{\tau}_{\alpha \beta}$ which glues together supermoduli space, let us consider what happens if we make different choices in this construction. Theorems 2.6 and 2.7 say that the difference in the choices should produce a map $\wedge \mathscr{E} \stackrel{\tau}{\longrightarrow} \wedge \mathscr{E}$. For simplicity, take the same open cover $\left\{\mathscr{V}_{1}, \mathscr{V}_{2}\right\}$ for $\pi^{-1}(\mathscr{U})$, but instead of $\phi_{1}, \ldots, \phi_{2 g-2}$, take a different set of sections $\psi_{1}, \ldots, \psi_{2 g-2} \in \Gamma\left(\mathscr{V}_{1} \cap \mathscr{V}_{2}, \kappa^{-1 / 2}\right)$, such that the cohomology class $\left[\theta^{a} \psi_{a}\right]$ is still the identity section of $\pi_{*}^{1}\left(\kappa^{1 / 2}\right) \otimes \mathscr{E}$. Then $\theta^{a} \psi_{a}=\theta^{a} \phi_{a}+\zeta_{1}-\zeta_{2}$, where $\zeta_{i}$ is holomorphic in $\mathscr{V}_{i}$. Then the super Riemann surfaces defined by $\exp \left(\theta^{a} \phi_{a}\right)$ and $\exp \left(\theta^{a} \psi_{a}\right)$ are isomorphic to first order in $\theta$. To see what happens to second order in $\theta$, we 
compute

$$
\begin{aligned}
& \exp \left(-\theta^{a} \phi_{a}\right) \exp \left(-\zeta_{1}\right) \exp \left(\theta^{b} \psi_{b}\right) \exp \left(\zeta_{2}\right) \\
& \quad=\exp \left(-\theta^{a} \phi_{a}\right) \exp \left(-\zeta_{1}\right) \exp \left(\theta^{a} \phi_{a}+\zeta_{1}-\zeta_{2}\right) \exp \left(\zeta_{2}\right) \\
& \quad=\exp \left(-\theta^{a} \phi_{a}-\zeta_{1}+\frac{1}{2}\left[\theta^{a} \phi_{a}, \zeta_{1}\right]\right) \exp \left(\theta^{a} \phi_{a}+\zeta_{1}+\frac{1}{2}\left[\theta^{a} \phi_{a}, \zeta_{2}\right]+\frac{1}{2}\left[\zeta_{1}, \zeta_{2}\right]\right) \\
& \quad=\exp \left(\frac{1}{2}\left(\left[\theta^{a} \phi_{a}, \zeta_{1}\right]+\left[\theta^{a} \phi_{a}, \zeta_{2}\right]+\left[\zeta_{1}, \zeta_{2}\right]\right)+o\left(\theta^{2}\right)\right)
\end{aligned}
$$

The section

$$
\frac{1}{2}\left(\left[\theta^{a} \phi_{a}, \zeta_{2}\right]+\left[\theta^{a} \phi_{a}, \zeta_{1}\right]+\left[\zeta_{1}, \zeta_{2}\right]\right) \in \Gamma\left(\mathscr{V}_{1} \cap \mathscr{V}_{2}, \wedge^{2} \mathscr{E} \otimes \kappa^{-1 / 2}\right)
$$

defines a cohomology class

$$
\chi \in H^{1}\left(\pi^{-1}(\mathscr{U}), \wedge^{2} \mathscr{E} \otimes \kappa^{-1 / 2}\right)=\wedge^{2} \mathscr{E} \otimes H^{0}\left(\mathscr{U}, \pi_{*}^{1}\left(\kappa^{-1 / 2}\right)\right) .
$$

The Kodaira-Spencer map identifies $\chi$ with a $\wedge^{2} \mathscr{E}$ valued vector field on $\mathscr{U}$. Then up to second order in $\theta, \wedge \mathscr{E} \stackrel{\tau}{\longrightarrow} \wedge \mathscr{E}$ is given by $1+\chi$. The higher order terms of $\tau$ may be computed in a similar fashion.

\section{Appendix. Does the Universal Curve over Level-n Spinmoduli Space Admit a Square Root of $\kappa$ ?}

Recall that $M_{n}=\widetilde{T} / \Gamma_{n}$, where $\widetilde{T}=($ Teichmüller space $) \times$ (spin structures), and $\Gamma_{n}$ is the level- $n$ subgroup of the mapping class group. Let $\sigma$ be the fiberwise spin structure described in Sect. $2, \sigma \in H^{0}\left(M_{n}, \tilde{\pi}_{*}^{1}\left(\mathbf{Z}_{2}\right) . \sigma\right.$ gives rise to a square root of $\kappa$ if and only if $0=d_{2} \sigma \in H^{2}\left(M_{n}, \mathbf{Z}_{2}\right)$. We do not know in general whether $d_{2} \sigma=0$, but we can offer the following observations. First note that $M_{n}$ is a disjoint union of two pieces, called the even and odd spinmoduli spaces, corresponding to the even and odd spin structures. (For a discussion of the parity of a spin structure see [At] or $[\mathrm{J}]$.)

Proposition 3.1. For $2 \leqq g \leqq 4, d_{2} \sigma$ vanishes on the odd spinmoduli space.

Proof. By results of $[\mathrm{E}-\mathrm{K}]$, for $g \leqq 4$ the universal curve over Teichmüller space admits exactly $2^{g}\left(2^{g}-1\right) / 2$ half-canonical divisors, one for each odd spin structure. Thus we can replace (Teichmüller space) $\times$ (odd spin structures) by (Teichmüller space $) \times$ (half-canonical divisors over the universal curve). It is then clear that $U \rightarrow\left(M_{n}\right)_{\text {odd }}$ admits a half-canonical divisor, which defines a square root of $\kappa$.

For higher genus, the problem is more difficult. Since $n \geqq 3, M_{n}$ is in fact a model for $B\left(\Gamma_{n}\right)$. Therefore $d_{2} \sigma$ can be described in terms of group cohomology. Fix a spin structure $\omega \in H^{1}\left(S_{\text {rel }} \Sigma, \mathbf{Z}_{2}\right)$ on the standard surface. Let $G \subset \operatorname{Diff} \Sigma$ be the subgroup fixing $\omega . \omega$ is an equivalence class of double covers of $S_{\mathrm{rel}} \Sigma$. If we choose a particular double cover $P \rightarrow S_{\text {rel }} \sum$ corresponding to $\omega$, then for $g \in G$, the fact that $d g^{*} \omega=\omega$ means that there are two isomorphisms of $P$ covering $d g$. Thus if we let $\tilde{G}$ be the group of transformations of $P$ covering $d g$ for some $g$, we get a central extension.

$$
0 \rightarrow \mathbf{Z}_{2} \rightarrow \widetilde{G} \rightarrow G \rightarrow 1,
$$

and $d_{2} \sigma$ vanishes on $M_{\text {(parity of } \omega)}$ if and only if this central extension splits when restricted to $G \cap \operatorname{Diff}_{n} \Sigma$. We can at least observe 


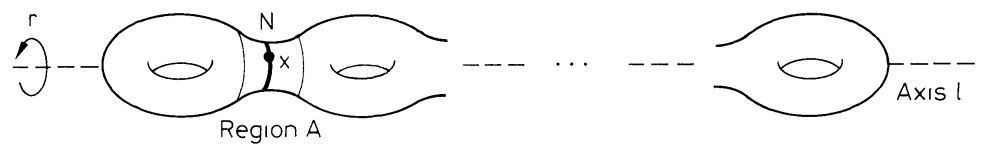

Fig. 3.1

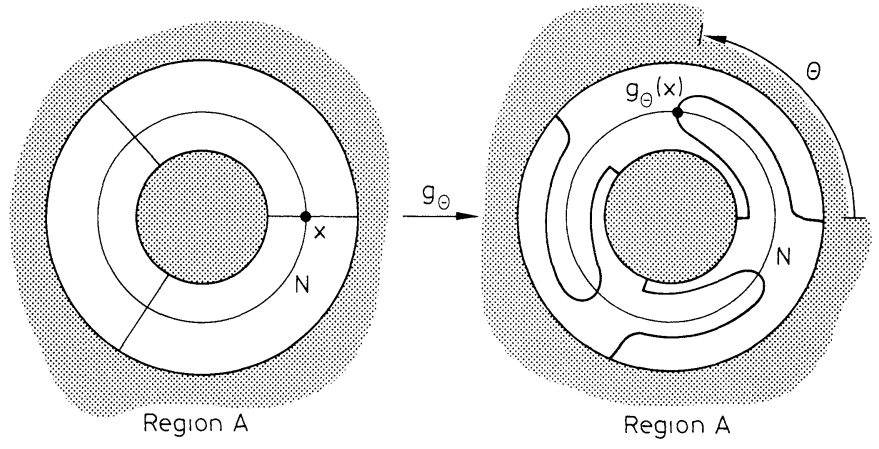

Proposition 3.2. The sequence $(*)$ does not split.

Proof. Certainly, Diff ${ }_{0} \Sigma \subset G$. Since Diff ${ }_{0} \Sigma$ is contractible, it is easy to see that (*) splits on Diff ${ }_{0} \Sigma$, and since every element of $\operatorname{Diff}_{0} \Sigma$ has a square root in $\operatorname{Diff}_{0} \Sigma$, there is a unique splitting $\operatorname{Diff}_{0} \Sigma \stackrel{v}{\longrightarrow} \widetilde{G}$. Now represent $\Sigma$ as in Fig. 3.1, so that rotation through $\pi$ about axis $l$ maps $\Sigma$ to itself. For $0 \leqq \theta \leqq 2 \pi$, let $g_{\theta}$ be the diffeomorphism which rotates neck $N$ through an angle $\theta$, imagining region $A$ to be made of rubber, and so that the complement of region $A$ remains fixed. Then $g_{2 \pi}$ fixes the point $x$ and the tangent vector $v$ of $N$. Thus $v\left(g_{2 \pi}\right): P \rightarrow P$ fixes the fiber over $v$.

Claim. $v\left(g_{2 \pi}\right)$ acts nontrivially on the fiber of $P$ over $v$.

Proof. Observe that the neck $N$ is homologically trivial. It follows that the loop $\left\{\theta \rightarrow d g_{\theta}(v)\right\} \subset S_{\text {rel }} \Sigma$ is homologically the fiber class. Since any spin structure is nontrivial on the fiber class, the claim follows.

To finish the proof of Proposition 3.2, let $h \in \operatorname{Diff} \Sigma$ be the diffeomorphism $h=r \cdot g_{\pi}$, where $r$ is rotation through $\pi$ about axis $l$. $r$ commutes with $g_{\pi}$ and $r^{2}=1$, so $h^{2}=g_{2 \pi}$. Moreover, $h$ acts by -1 on homology, and so fixes all spin structures. Also, $h$ fixes the point $x$ and the vector $v$. So if there were a splitting, $\tilde{v}$, of $(*)$, on any subgroup of $G$ containing $\operatorname{Diff}_{0} \Sigma$ and $h, v\left(g_{2 \pi}\right)$ would act on the fiber of $P$ over $v$ by $\tilde{v}(h)^{2}$, which is trivial. This contradicts the claim and finishes the proof.

Acknowledgements. The authors would like to thank Pierre Deligne, Irwin Kra, the Institute for Advanced Study, and the anonymous referee.

\section{References}

[A] Ahlfors, L.: The complex analytic structure of the space of closed Riemann surfaces. In Analytic functions, pp. 349-376. Princeton, NJ: Princeton University Press 1960

[At] Atiyah, M.: Riemann surfaces and spin structures. Ann. Sci. Ec. Norm. Super., Ser. 4, t. 4, 47-62 (1971) 
[B-E] Bers, L., Ehrenpreis, L.: Holomorphic convexity of Teichmüller spaces. Bull. AMS 70, $761-764$ (1964)

[C-R] Crane, L., Rabin, J.M.: Super Riemann surfaces: uniformization and Teichmüller theory. Commun. Math. Phys. 113, 601-623 (1988)

[E-K] Earle, C.J., Kra, I.: Half-canonical divisors on variable Riemann surfaces. J. Math. Kyoto Univ. 26, 39-64 (1986)

[E-L] Eastwood, M., Le Brun, C.: Thickenings and supersymmetric extensions of complex manifolds. Am. J. Math. 108, 1177-1192 (1986)

[F-K] Farkas, H., Kra, I.: Riemann surfaces. Graduate texts in mathematics, Vol. 71. Berlin, Heidelberg, New York: Springer 1980

[Fr] Freidan, D.: Notes on string theory and two dimensional conformal field theory. EFI preprint $85-99$

[G] Grothendieck, A.: Construction de l'espace de Teichmüller. Sem. Henri Cartan 13 (1961) exposé 17

[J] Johnson, D.: Spin structures and quadratic forms on surfaces. J. Lond. Math. Soc. (2), 22, 365-373 (1980)

[K-S] Kodaira, K., Spencer, D.C.: On deformations of complex analytic structures. I, II. Ann. Math. 67, 328-466 (1958); II. Ann. Math. 71, 43-76 (1960)

[M] Manin, Yu.I.: Critical dimensions of the string theories and the dualizing sheaf on the moduli space of (super) curves. Funct. Anal. Appl. 20, 244245 (1987)

[R] Rothstein, M.: Deformations of complex supermanifolds. Proc. AMS 95, 255-260 (1985)

Communicated by L. Alvarez-Gaumé

Received July 4, 1987; in revised form December 15, 1987 